\title{
ESTUDIO DE LA OFERTA DE PRECIOS HOTELEROS ANTICIPADA A TRAVÉS DE UN IMPORTANTE CANAL DE DISTRIBUCIÓN ONLINE
}

\author{
Gloria Sánchez-Lozano* \\ Universidad de Sevilla \\ https://orcid.org/0000-0002-9403-7570 \\ Luis Nobre Pereira** \\ Universidade do Algarve \\ https://orcid.org/0000-0003-0917-7163 \\ Esther Chávez-Miranda*** \\ Universidad de Sevilla \\ https://orcid.org/0000-0002-6170-3514
}

\section{RESUMEN}

Este trabajo se centra en el estudio de la oferta de precios a través de canales online (Booking.com) en un destino turístico. Los hoteles se han seleccionado mediante muestreo aleatorio simple estratificado con afijación proporcional. Se trabaja con un conjunto de datos de grandes dimensiones (big data, con un total de 1.353.751 registros). Se considera una antelación de estancia de aproximadamente un año, lo que supone una gran novedad respecto a estudios previos. En el trabajo se presentan los valores de precios considerando los datos habitualmente publicados por los hoteles en estos canales, lo que permite ofrecer una panorámica de la operativa relativa a la oferta de precios a través de la distribución online a nivel de destino turístico.

Palabras clave: Pricing; canales de distribución online; gestión de canales de distribución; antelación; revenue management; hotel.

Fecha de recepción: 8 de marzo de 2019

Fecha de aceptación: 15 de enero de 2020

* Departamento de Economía Financiera y Dirección de Operaciones. Facultad de Ciencias Económicas y Empresariales. Universidad de Sevilla. Avda. Ramón y Cajal, 1. 41018 SEVILLA (España). E-mail: gsanchez7@us.es

** Escola Superior de Gestão, Hotelaria e Turismo \& Centro de Investigação em Turismo, Sustentabilidade e Bem-estar. Universidade do Algarve. Campus da Penha. 8005-139 FARO (Portugal).E-mail: Imper@ualg.pt

*** Departamento de Economía Financiera y Dirección de Operaciones. Facultad de Turismo y Finanzas. Universidad de Sevilla. Avda. San Francisco Javier, s/n. 41018 SEVILLA (España).E-mail: revenue@ us.es 


\title{
Study of hotel prices in advance through a major online distribution channel
}

\begin{abstract}
This work focuses on the study of prices offered through an online distribution channel (Booking.com) on a concrete tourism destination. Hotels were selected by a stratified simple random sampling method with proportional allocation. Research is done over an extensive dataset $(1,353,751$ of records, big data). The advance booking period considered covers approximately a whole year, and this represents a great novelty with respect to previous studies. Average prices are calculated from the values ordinarily published by hotels on this type of channels, which allows providing the outline of the operational management of prices implemented on the destination, through the online distribution and from the supply perspective.
\end{abstract}

Keywords: Pricing; online distribution channels; channel management; booking horizon; revenue management; hotel.

\section{IMPORTANCIA DEL ESTUDIO DE PRECIOS OFERTADOS CON ANTELA- CIÓN EN CANALES ONLINE}

Conforme a su definición clásica, revenue management en el sector hotelero persigue la asignación del precio correcto al cliente correcto, en el momento correcto y a través del canal apropiado, de forma que esta combinación proporcione a la empresa el máximo beneficio y/o ingreso posible con sus recursos disponibles. El "momento" constituye un elemento esencial e inequívoco de la aplicación de revenue management y es de singular importancia. De un lado, permite la comercialización anticipada de los recursos y el establecimiento de las condiciones en que estos se ofertan, pero, de otro, incrementa la complejidad de su gestión porque el número de decisiones a adoptar se multiplican de forma notable.

Así, por ejemplo, en el sector hotelero, el recurso (o unidad de inventario "habitación por noche") y los elementos relacionados con este (precio, tipo de habitación, número de huéspedes alojados por habitación y longitud de la estancia) se pueden ofertar de forma integrada con un año de antelación, con el propósito de recibir peticiones de clientes hasta el momento en que se preste el servicio. La persona encargada de esta gestión (por lo general, el revenue manager) deberá establecer una estrategia y desarrollar las tácticas adecuadas durante todo este tiempo para mejorar los niveles de rentabilidad, lo que plantea como necesidad conocer cómo evoluciona la oferta de precios dependiendo de los atributos que la definen a lo largo de todo el período de antelación.

Esta investigación estudia las políticas de precios aplicadas en un canal de distribución hotelera online. La fijación de precios (pricing) se considera un elemento básico y de gran relevancia en materia de revenue management (Weatherford y Bodily, 1992; McGill y Van Ryzin, 1999, Baker y Collier, 1999; Chiang, Chen y Xu, 2007; Ropero, 2011; Guillet y Mohammed, 2015), mientras que la gestión de canales de distribución, constituye un área 
de investigación relativamente nueva que ha ido ganando importancia más recientemente, especialmente dentro del contexto hotelero (Ivanov y Zhechev, 2012; Guillet y Mohammed, 2015; Domingo-Carrillo et al., 2017). Además, el panorama de la distribución hotelera ha sufrido una gran transformación derivando en la necesidad de desarrollo de investigaciones centradas en su estudio (Law, Leung, Lo, Leung y Fong, 2015).

Internet ha cambiado de forma radical el modo en que los profesionales del sector hotelero solían comunicar y compartir los precios por noche para sus habitaciones disponibles, aunque, al mismo tiempo, se han tenido que asumir nuevos costes (Vinod, 2004). Por ello, en la actualidad, resulta cada vez más necesario llevar a cabo una gestión eficiente de los canales de distribución en un escenario complejo (Kimes, 2016) que requiere tanto incrementar el tiempo dedicado a estas actividades como considerar los costes atribuibles a las mismas. Las actividades de seguimiento y control en un entorno cambiante y competitivo deben considerarse (Josephi, Stierand y Van Mourik, 2016). Pese a las razones mencionadas, la importancia de la gestión de canales y, su influencia en los costes y en los indicadores de revenue management, existen carencias en investigación a este respecto, sobre todo desde el punto de vista de la oferta.

En el marco de la comercialización online, Booking.com está reconocido como uno de los principales canales de distribución hotelera. Junto con Expedia, se erige como el mayor impulsor de los cambios del sector. Booking.com destaca por su papel principal según sus cifras de ventas crecientes y su adopción y avances en las nuevas tecnologías, como, por ejemplo, las encaminadas a realizar reservas a través de terminales móviles. Para un hotel, el número de reservas realizadas a través de esta agencia de viajes online (OTA, por sus siglas en inglés -Online Travel Agency) en Europa puede suponer el 60\% del total de sus ventas (Tom Dieck, Fountoulaki y Jung, 2018). En términos generales, los beneficios de la comercialización online son múltiples considerando que es un sector con gran crecimiento en la actualidad y que permite multiplicar las posibilidades de acceso y comunicación con los clientes (Law et al., 2015).

El análisis de los precios y otras variables asociadas con la oferta hotelera online considerando un tiempo de antelación amplio, ofrece la posibilidad de anticiparse al panorama general que se plantea en el canal para un día concreto (en la fecha de check-in) y puede influir de forma notable en la generación de ingresos. Los revenue managers, en particular, y los directivos y gestores de hoteles, en general, pueden sacar provecho de esta visión general anticipándose a la situación y consiguiendo mejorar sus cifras de ventas.

Teniendo en cuenta lo anterior, esta investigación se plantea como propósito el estudio de los precios ofertados por los hoteles en un destino urbano a través de una agencia de viajes online (OTA) considerando una antelación de 300 días. En concreto se analizan los precios medios, máximos y mínimos publicados en función del perfil del hotel (categoría oficial y número de habitaciones) y de los atributos que acompañan al precio en cada oferta (día de la semana, tipo de habitación, número de personas alojadas por habitación (pax) y longitud de estancia). De forma adicional, el estudio se completa con la obtención de funciones de precios que mejor se ajustan a los datos observados.

Desde el punto de vista académico, se ofrece un soporte teórico y práctico que atiende conjuntamente a dos elementos o variables primordiales en la aplicación de revenue management: precios y antelación en su oferta. Un mejor conocimiento del mercado 
online hará posible adoptar decisiones que favorezcan mejores niveles de rentabilidad. Este estudio aplicado en un destino urbano nos permite la obtención de precios medios y su evaluación en este contexto. Asimismo, se establecen los primeros pasos encaminados al análisis conjunto de los precios y sus atributos considerando un amplio horizonte temporal de antelación.

A los profesionales del sector les permitirá contar con una referencia de los valores de precios medios, no solo como variable única, sino como variable dependiente de las características que la acompañan en su oferta integrada a lo largo de todo el período de antelación. Las funciones de precios permitirán, además, comprobar si los publicados por cada hotel en el destino analizado o respecto a este son similares a los de la plaza en la que opera.

La rigurosidad en el proceso de selección de la muestra (muestreo aleatorio simple estratificado con afijación proporcional), el volumen de datos considerados (1.353.751 registros) y la amplitud del período de estudio considerado (antelación de 300 días) son valores añadidos de la investigación realizada que, además, garantizan la posibilidad de extrapolación de resultados en el contexto analizado. Los resultados, además, pueden constituir una referencia para el desarrollo de futuras investigaciones a nivel de destino turístico.

\section{METODOLOGÍA}

\subsection{Destino, población y muestra objeto de estudio}

El destino escogido es Sevilla, una ciudad situada en el sur de España y conocida mundialmente por su patrimonio cultural. Se selecciona por su importancia relativa en comparación con otras ciudades de España, ya que presenta una posición predominante. Desde hace años ostenta uno de los primeros puestos (entre el tercero y el sexto) del ranking de destinos urbanos de España, según los diversos informes presentados por Exceltur (2013, 2016, 2017 y 2018). Recientemente, ha sido galardonada con la primera posición en la categoría de ciudades en la clasificación de Lonely Planet (2018), hecho que viene a confirmar su relevancia como destino urbano.

Con respecto al perfil elegido para los hoteles, en primer lugar, aclarar que el sistema oficial en España clasifica a los hoteles en cinco categorías diferentes, otorgándole un número de estrellas inferior o superior acorde con la calidad de las instalaciones y la variedad de los servicios prestados (de menor a mayor, de una a cinco estrellas). En España, las investigaciones anteriores realizadas en materia de revenue management se centran en las categorías más altas, de tres a cinco estrellas o, al menos, en hoteles de cuatro y cinco estrellas (Chávez-Miranda, 2005; Talón-Ballestero, 2010; Oteo-Iturmendi, 2013; Domingo-Carrillo, 2016). En este caso, se ha trabajado en línea con estas investigaciones previas, incluyendo también hoteles de las tres categorías más altas.

Antes de poder seleccionar una muestra representativa de hoteles, fue necesario identificar los elementos de la población. Para ello se utilizó como referencia el censo de hoteles que proporcionaba el "Informe Anual de Hostelería Hostelmarket 2014" que se comparó y completó con los hoteles que listaba Booking.com cuando se hacía una petición de reserva en Sevilla ciudad. Una vez que la población total se estableció con seguridad, el 
tamaño muestral requerido fue calculado por muestreo aleatorio simple (5\% error, $95 \%$ nivel de confianza, $\mathrm{p}=\mathrm{q}$ ).

El censo de Hostelmarket mencionado anteriormente presentaba la información necesaria sobre la categoría oficial (número de estrellas) de los hoteles, lo que nos permitió dividir la muestra total estableciendo tres estratos, uno por cada categoría de hotel. Los individuos (hoteles) de la muestra que debían ser asignados a cada uno de los estratos se determinó mediante muestreo aleatorio simple estratificado con afijación proporcional, es decir, manteniendo en cada estrato el peso relativo de esa subpoblación con respecto a la población total. La selección de la muestra de hoteles de cada categoría se obtuvo de forma aleatoria utilizando el software estadístico IBM SPSS Statistics (versión 24). En Sevilla, 94 hoteles componían la población total porque cumplían con los requisitos del perfil de hotel definido para el estudio. Están localizados en la ciudad de Sevilla (no en Sevilla Provincia) y se clasifican en hoteles de tres (38 hoteles), cuatro (51 hoteles) y cinco estrellas (5 hoteles). Por lo tanto, la afijación proporcional resultante para cada uno de los estratos de la muestra (31 hoteles) es la siguiente: tres estrellas (12 hoteles), cuatro estrellas (17 hoteles) y cinco estrellas ( 2 hoteles). Este método de muestreo complejo asegura que los resultados y sus conclusiones puedan ser extrapolados tanto a nivel de categoría de hotel como a nivel de Sevilla como destino urbano.

\subsection{Datos y variables analizadas}

Los datos pertinentes para el estudio se recopilaron de entre la información publicada por estos hoteles en Internet, concretamente en la web de Booking.com mediante búsquedas automatizadas. Dichas consultas se realizan en una fecha concreta, el 20 de enero de 2017 (día 0, mismo día de la extracción), y cubren los 300 días siguientes a la fecha de extracción (es decir, hasta el 16/11/2017). La información se refiere a los datos publicados por cada uno de los hoteles de la muestra para cada uno de los días incluidos en este período. Este proceso resulta en un conjunto de datos de gran volumen, en una cantidad tal que puede considerarse big data.

En términos generales, se utiliza la variable precios como el principal objeto de estudio. En cuanto a las variables adicionales disponibles a continuación se describe su naturaleza y medición. (1) Categoría de hotel contiene información acerca del número oficial de estrellas de los hoteles de la muestra; varía entre tres y cinco estrellas, ambos inclusive. (2) Número de habitaciones proporciona información acerca de la dimensión física o capacidad disponible de los hoteles; presenta valores extremos, entre 7 y 437 habitaciones. (3) Día de la semana es una variable temporal que se refiere al día de la semana para el cual un precio se ha ofertado; se ha codificado conforme a valores numéricos partiendo de $1=$ domingo a $7=$ sábado). La variable (4) tipo de habitación, debido a la gran variabilidad que presentaban los valores recopilados se recodifica en grupos homogéneos que incluyen las categorías: "estándar", "superior", "superior plus", "junior suite" y "suite". (5) Número de huéspedes se refiere, como su nombre indica, al número de ocupantes máximo que se pueden alojar una habitación; los valores que presenta se comprenden entre 1 y 4 personas. (6) Longitud de la estancia contiene información sobre el número de noches para el cual se ha configurado y publicado un 
precio por noche concreto; puede variar de 1 a 4 noches. Respecto a esta variable es importante aclarar que los precios que se exponen vienen referidos al importe aplicado por noche y, por tanto, no se presentan los valores para el total de la estancia. Por ejemplo, una tarifa de $177 €$ con una longitud de estancia de 2 noches se refiere al precio que se aplicaría cada una de las noches de estancia y, por tanto, para calcular el importe total de la reserva se debe multiplicar este por dos.

De este conjunto de variables, la número (4) tipo de habitación, como ya se había adelantado, es una variable recodificada. Los hoteles de Sevilla ofertan sus disponibilidades bajo más de 80 denominaciones diferentes para identificar las habitaciones puestas a la venta, lo que nos lleva a que, a veces, habitaciones prácticamente idénticas en cuanto a sus atributos físicos se están comercializando con denominaciones diferentes. Estos nombres posibles se redujeron y clasificaron bajo las nuevas cinco categorías que se usan en el estudio (estándar, superior, superior plus, junior suite y suite) atendiendo básicamente a dos criterios que consideramos principales: dimensión probable de la habitación y su estilo, amenities y servicios incluidos).

\subsection{Acerca de los datos y su explotación}

El conjunto de datos contiene 193.393 líneas por cada una de las variables analizadas (siete, incluyendo la variable dependiente, precio), esto resulta en 1.353.751 registros y no se observan valores perdidos. Hay que destacar como una muestra relativamente pequeña de hoteles en Sevilla ciudad (31) puede producir una oferta de precios tan numerosa, en tan sólo uno de los canales de distribución online con los que probablemente trabajan.

Los resultados se muestran de dos formas en conjunción con los objetivos del estudio: (a) precio medio de la habitación, el cual se calcula directamente a partir de la variable "precio" para los 300 días previos a la llegada de clientes y (b) precios máximos y mínimos observados por cada serie de datos considerando también la totalidad del período de antelación.

El procesamiento de los datos, las tablas y las figuras se han obtenido con MS Excel (versión 2016).

Para la obtención de la función de precios, dependiendo de cada una de las variables del estudio, que mejor se ajusta a los datos disponibles se comparan los resultados $\left(\mathrm{R}^{2}\right)$ obtenidos conforme a una línea de tendencia lineal, exponencial, logarítmica y polinómica proporcionados por MS Excel (versión 2016) y se incluye en los resultados aquella que reporta un valor de ajuste más elevado. En cada uno de los apartados del trabajo se incorpora su expresión junto con el valor que debe tomar cada variable independiente (x, en la expresión) para poder obtener el precio medio correspondiente.

Para mostrar de forma gráfica la variedad de categorías que emplean los hoteles a la hora de publicar sus datos de oferta se proporciona una nube de palabras. Se obtiene a partir de cada una de las palabras que incorporan las 87 expresiones distintas identificadas. En este tipo de representación el tamaño de cada fuente nos aporta información acerca de la frecuencia con que se emplea cada término. Para su obtención se ha utilizado la aplicación Microsoft Power BI Desktop. 


\section{RESULTADOS}

\subsection{Categoría de hotel (número de estrellas)}

Considerando un horizonte de reservas de 300 días de antelación con respecto a la fecha de registro del cliente (check-in), los datos presentan un precio medio general de $177,8 €$ para Sevilla ciudad en el canal de distribución hotelera Booking.com. La figura que se muestra a continuación presenta los precios medios, máximos y mínimos por categoría de hotel (número de estrellas, de tres a cinco).

\section{Figura 1}

\section{PRECIOS MEDIOS, MÁXIMOS Y MÍNIMOS POR CATEGORÍA DE HOTEL}

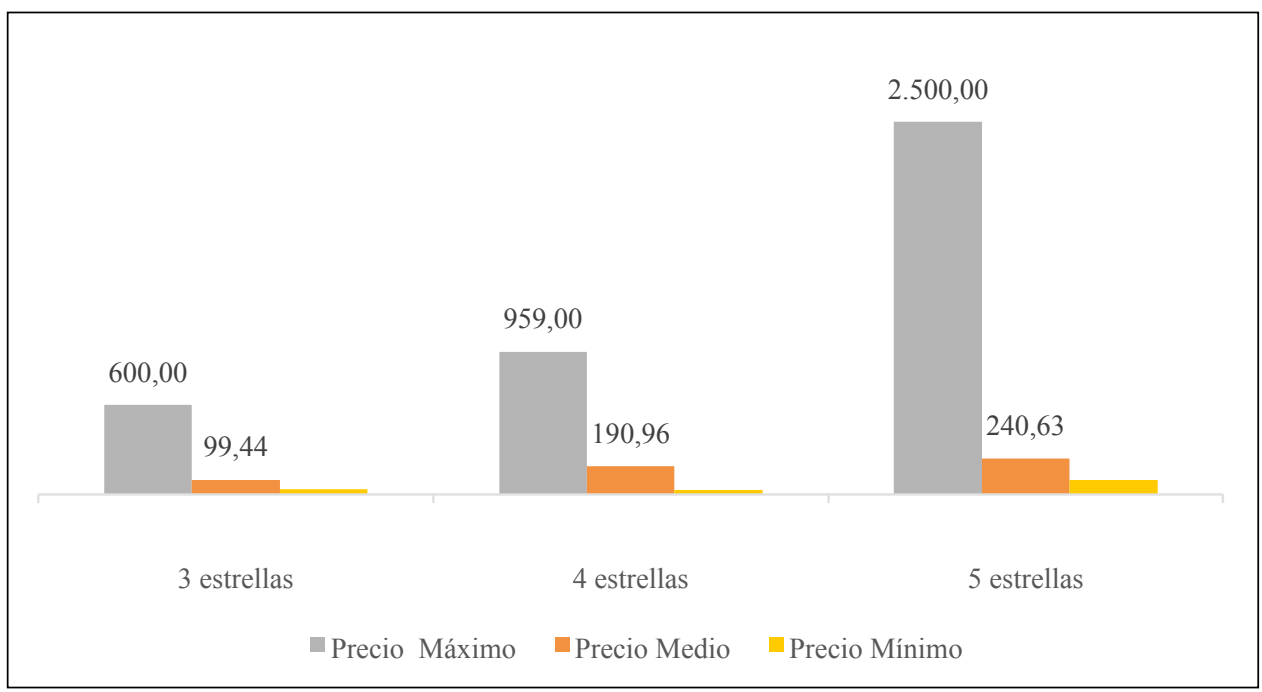

El precio medio se comporta de la forma esperada con respecto a la categoría de hotel, ya que aumenta para categorías más altas. Sin embargo, la subida que experimentan los precios medios no es proporcional: el valor medio para los hoteles de cuatro estrellas casi duplica el correspondiente a los de tres $(92,0 \%)$. No obstante, el incremento que experimenta el precio medio para los hoteles de cinco estrellas en comparación con el de cuatro es de tan sólo un 26.0\%. El aumento medio entre categorías de hotel es de 70,6€ $(41.3 \%)$, superior al correspondiente a la industria hotelera polaca, el cual se registra entre el +30,0-36,0\% (Pawlicz y Napierala, 2017). Los hoteles con una categoría más alta, a su vez presentan precios extremos más diferentes entre sí. El rango entre los valores mínimos y máximos es muy amplio, con diferencias de 566,1€, en los hoteles de tres estrellas y de hasta de $2.405,5 €$, en los de cinco.

Cabe resaltar que, a veces, los hoteles de tres estrellas aplican precios que podrían ser considerados más adecuados para los de cuatro. Tanto los hoteles de tres como de cuatro 
estrellas fijan precios que comienzan en valores mínimos similares $(33,9 €$ y $32,4 €$, respectivamente) y, por ejemplo, los precios máximos ofrecidos por hoteles de tres estrellas llegan a alcanzar valores de hasta $600,0 €$. En el mismo sentido, también se dan casos en los que hoteles de cinco estrellas ofrecen habitaciones a un precio de $94,5 €$, que podría considerarse como una tarifa más propia de un hotel de cuatro estrellas, o incluso de tres, dependiendo del tipo de habitación aparejado.

Se constata (Expresión 1) que el comportamiento de los precios medios con respecto a la categoría de hotel se aproxima a una función logarítmica, $\left(\mathrm{R}^{2}=0,9996\right)$. A medida que el número de estrellas se eleva, el precio medio también. La siguiente función estimada presenta esta relación, donde y se refiere al precio medio y $\mathrm{x}$ a la categoría de hotel $(\mathrm{x}=1$, para tres estrellas; $\mathrm{x}=2$, para cuatro estrellas; $\mathrm{x}=3$, para cinco estrellas).

$$
\mathrm{y}=128,9 \ln (\mathrm{x})+100,02 .
$$

(Expression 1)

\subsection{Número de habitaciones}

En este apartado, el precio medio se estudia en conjunto con el número de habitaciones que tienen cada uno de los hoteles incluidos en la muestra (Figura 2). Es necesario aclarar que algunos de los precios medios que se muestran en el gráfico, se han obtenido considerando los precios ofertados por más de un hotel. Este hecho se debe a que algunos hoteles tienen exactamente el mismo número de habitaciones (hay dos hoteles de siete habitaciones, dos hoteles de 23 habitaciones y otros dos de 81 habitaciones).

\section{Figura 2 \\ PRECIOS MEDIOS MÁXIMOS Y MÍNIMOS POR NÚMERO DE HABITACIONES}

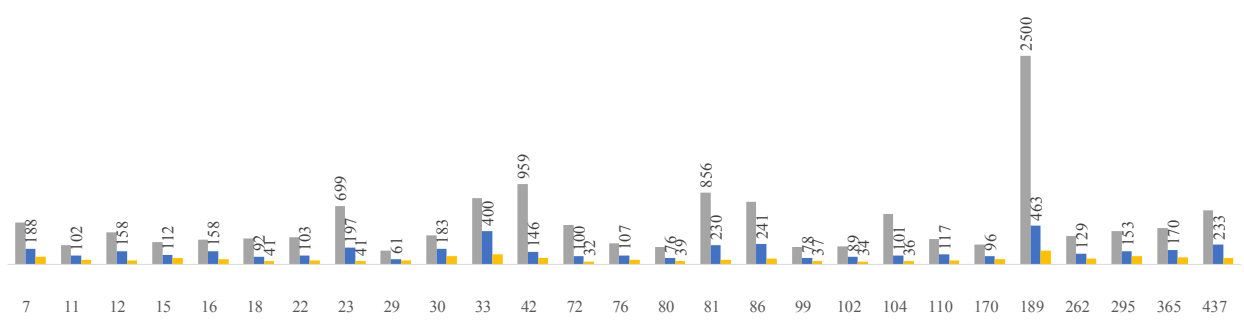

Como puede observarse, los precios medios no parecen presentar un patrón concreto de comportamiento con respecto al número de habitaciones del hotel. No obstante, si se analizan los datos en detalle, se pueden identificar algunos casos especiales, los cuales se describirán más tarde en este apartado. En general, los precios medios más altos se corresponden con hoteles de 33 y 189 habitaciones. Se trata en ambos casos de hoteles de cuatro estrellas, aunque difieren sustancialmente en el número de habitacio- 
nes (156). Por ejemplo, vemos que la influencia del número de habitaciones no sigue una pauta cuando comprobamos que el hotel de menor número de habitaciones (siete), las está comercializando a un precio medio superior que un hotel que cuenta con 365 habitaciones.

El gráfico anterior también nos permite identificar los hoteles que en términos generales aplican las tarifas más altas y más bajas en el destino (considerando hoteles de 3, 4 y 5 estrellas). Respecto a los valores máximos, los hoteles con 189, 42 (2 casos), 81 y 33 habitaciones destacan en su política de tarifas frente a los demás con valores de $2500 €$, $959 €, 856 €$ y $795 €$, respectivamente. Mientras que los valores mínimos, con precios inferiores a $40.00 € /$ noche (entre $32.40 €$ y decreciendo hasta $39.36 €$ ), se presentan en 5 casos $(72,102,104,99$ y 80$)$.

\subsection{Día de la semana}

Continuando con nuestro análisis, el propósito de este apartado es el de comprender la relación entre los precios medios y el día de la semana en que están disponibles para su contratación. Se constata que los precios medios se comportan de manera diferente dependiendo del día de la semana: son más altos durante los fines de semana y más bajos entre semana (Figura 3).

\section{Figura 3}

\section{PRECIOS MEDIOS, MÁXIMOS Y MÍNIMOS POR DÍA DE LA SEMANA}

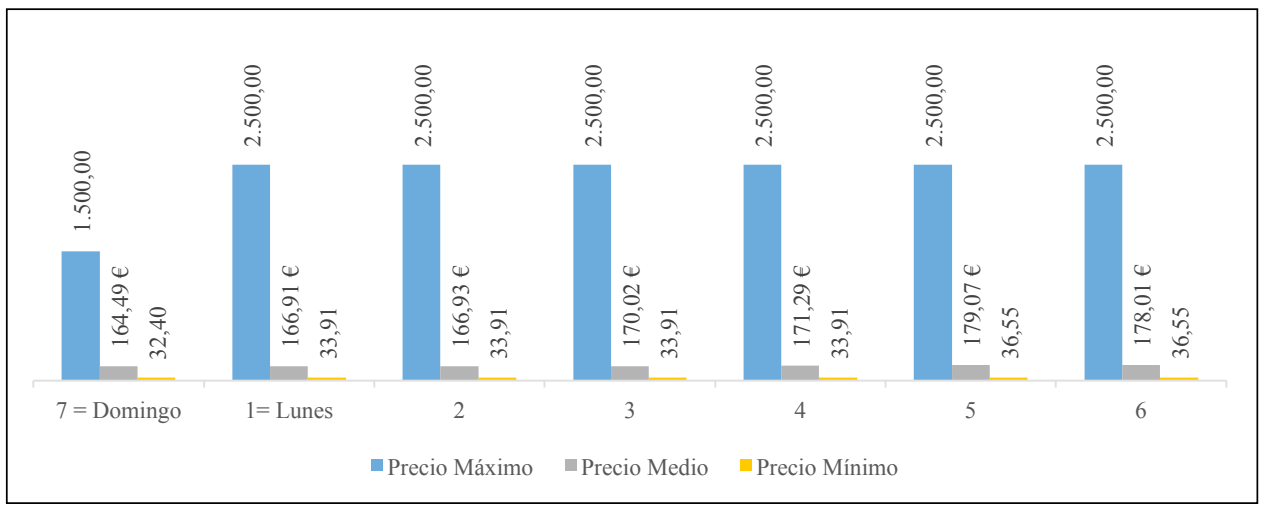

Tomando como referencia un nivel de precio de $175 €$, de entrada, podremos identificar dos tarifas diferentes. El precio medio ofertado durante el fin de semana es de 178,5€ (incluye los valores 5 y 6) y el precio medio de los días entre semana es de $167 €$ (incluye los valores 7, 1, 2, 3 y 4) con una diferencia de $10.6 €$ entre ambos. Un precio medio más elevado durante el fin de semana está razonablemente justificado, porque los usuarios principales de Booking.com son turistas que viajan por motivos vacacionales y de ocio (Law et al., 2015). 
Si continuamos analizando en mayor profundidad, podemos identificar una tarifa adicional durante la mitad de la semana, lo que nos llevaría a diferenciar tres grupos de precios: (1) comienzo de la semana, (2) mitad de la semana y (3) fin de semana. Los precios medios van subiendo progresivamente durante el miércoles y el jueves, justo antes del incremento más pronunciado de los viernes, que representa en este caso, el comienzo del fin de semana.

Podemos afirmar que los hoteles de Sevilla tienden a fijar precios más altos durante el fin de semana (para las noches del viernes y del sábado). Por el contrario, el precio medio más bajo corresponde al domingo, el cual marca el comienzo de la semana laboral.

En cuanto a los precios mínimos y máximos que se ofertan, se observa que toman valores exactamente iguales en la mayoría de los casos, independientemente del día de la semana que se está considerando (precio mínimo de 33,9€ y máximo de 2.500,0€). Este hecho nos hace pensar que se trata de los mismos hoteles concretos que están publicando precios extremos, y que están usando precios estáticos a lo largo de la semana.

Si observamos el recuento de precios que presentan los diferentes días de la semana, se puede comprobar que constan de un volumen de oferta similar independientemente del día de que se trate (entre 14,0\% y 14,6\%).

Los precios medios observados según el día de la semana se ajustan a una función polinómica de orden 2 (Expresión 2), $\mathrm{R}^{2}=0$,9164. El modelo estimado es el siguiente:

$$
y=0,2113 x^{2}+0,7828+163,6, \quad \text { (Expresión 2) }
$$

donde $\mathrm{x}$ se refiere al día de la semana (1=domingo y 7= sábado).

\subsection{Tipo de habitación}

En relación con esta variable los resultados obtenidos muestran la gran variedad de denominaciones empleadas por los hoteleros a la hora de comercializar sus productos en este canal de distribución. Se identifican un total de 87 expresiones distintas. En la Figura 4 se pueden observar de forma gráfica las distintas palabras empleadas y, dependiendo del tamaño de la fuente, la frecuencia en que son empleadas. A mayor frecuencia, mayor dimensión de la fuente. La nube nos permite identificar términos directamente relacionados con el tipo de habitación (double, twin, executive, suite) pero también anotados con vistas a hacer más atractiva la oferta (con jacuzzi, balcony, terrace, parking) e incluso que hacen referencia al número o personas a las que se dirige (2, triple, quadruple, child).

En la Figura 5 pueden observarse los precios (medios, máximos y mínimos) una vez realizada la reclasificación de habitaciones conforme a las categorías: standard, superior, superior plus, junior suite y suite. Como puede observarse, los precios medios muestran un comportamiento lógico según el tipo de habitación a la venta. Éstos suben a medida que observamos una categoría de habitación superior. 


\section{Figura 4}

NUBE DE TÉRMINOS UTILIZADOS PARA DISTINTOS TIPOS DE

\section{HABITACIÓN}

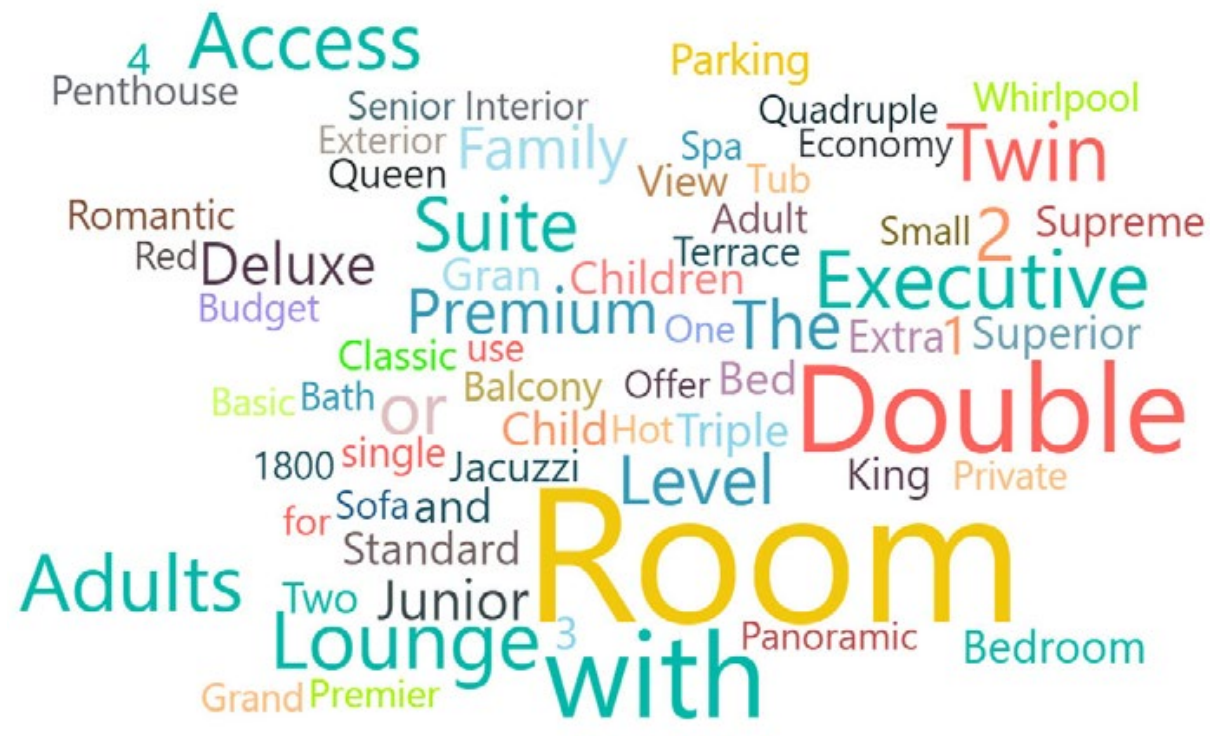

Figura 5

PRECIOS MEDIOS, MÁXIMOS Y MÍNIMOS POR TIPO DE HABITACIÓN

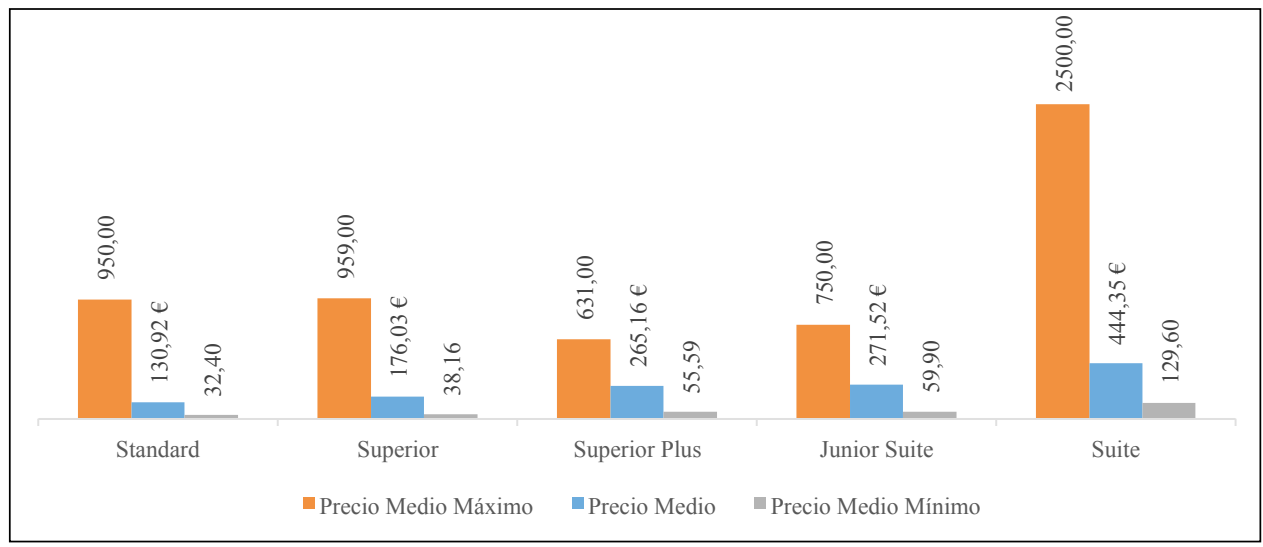


El precio medio aumenta de forma progresiva y el incremento medio que correspondería a cada salto de categoría es de 78,4€. Sin embargo, en dos casos el incremento es más pronunciado y aumenta más que la media correspondiente. Esto ocurre en el paso de tipo de habitación superior a superior plus $(89,1 €)$ y el relativo de junior suite a suite $(172,8 €)$. De otra parte, la menor de las subidas se registra en el cambio de tarifas de superior plus a junior suite $(6,4 €)$.

Los dos saltos más pronunciados en los precios medios se explican por grandes diferencias, bien en la dimensión de las habitaciones clasificadas en esa tipología de habitación (superior plus a veces presenta espacios y servicios similares a apartamentos) o bien por un cambio en la calidad de los servicios proporcionados (servicios premium en el caso de las suites). Las habitaciones clasificadas como superior plus y junior suite van unidas a tamaños y servicios similares y, por tanto, se están ofreciendo a precios medios muy parecidos.

Los precios mínimos aplicados también presentan unos valores lógicos, incrementándose de acuerdo con los cambios de tipo de habitación. Por el contrario, los precios máximos no siguen esta pauta. Alcanzan un valor de 950,0€ para las habitaciones de tipo estándar y superior, mientras que para los tipos de habitación superior plus y junior suite, los precios máximos publicados son de $631,0 €$ y de $750,0 €$, respectivamente.

La tipología de habitación que presenta el rango más estrecho de precios es superior plus (tiene una amplitud de $575,4 €$ de diferencia entre el precio mínimo y el máximo registrado) en comparación con el resto de las categorías: estándar $(917,6 €)$, superior (920,8€), junior suite $(690,1 €)$ y suite $(2.370,4 €)$.

Acerca del volumen de oferta relativo a cada tipo de habitación, podemos resaltar que más del $85 \%$ de los precios ofertados comercializan habitaciones de tipo estándar o superior $(52,1 \%$ y $33,0 \%$, respectivamente).

Finalmente, queda comprobado que el comportamiento de los precios medios con respecto al tipo de habitación se aproxima a una función exponencial (Expresión 3), $\mathrm{R}^{2}=0,9552$. El modelo estimado que se presenta a continuación muestra el comportamiento creciente de los precios a medida que la dimensión y los servicios incluidos en las habitaciones son mayores o presentan una calidad superior,

$$
\mathrm{y}=99,687 \mathrm{e}^{0.2877 \mathrm{x}}, \quad \text { (Expresión 3) }
$$

donde $\mathrm{x}$ se refiere al tipo de habitación ( $\mathrm{x}=1$ si estándar; $\mathrm{x}=2$ si superior; $\mathrm{x}=3$ si superior plus; $x=4$ si junior suite; $x=5$ si suite).

\subsection{Número de huéspedes}

Los precios medios por habitación se comportan de manera ascendente con respecto al número máximo de huéspedes a alojar (Figura 6). Pese a esta tendencia creciente, el incremento en cada nivel de precios no es proporcional a un número superior de huéspedes. 


\section{Figura 6 \\ PRECIOS MEDIOS, MÁXIMOS Y MÍNIMOSPOR NÚMERO DE HUÉSPEDES}

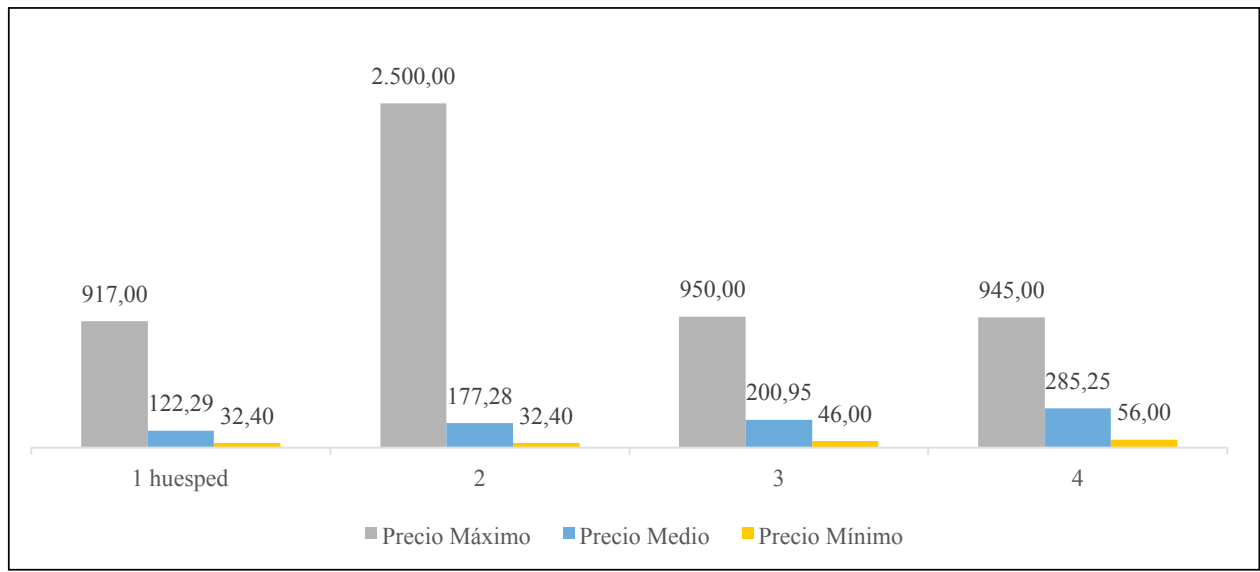

El incremento medio esperado para cada huésped adicional es de $40,7 €$. Esta cifra nos permite estudiar la magnitud de las subidas que experimenta el precio medio para capacidades de habitación superiores. En el primer cambio de uno a dos huéspedes, el precio medio está creciendo más que proporcionalmente $(54,9 €)$. En el segundo salto, de dos a tres huéspedes, se observa el incremento más leve $(23,7 €)$ e inferior al esperado. Esto puede deberse a una práctica común del sector hotelero, por la que se suele instalar una cama adicional en las habitaciones dobles más espaciosas con el propósito de comercializarlas como habitaciones triples, aunque en realidad se trate del mismo espacio físico que se está ofertando a un precio ligeramente superior. Por último, en el cambio de tres a cuatro huéspedes, el precio medio experimenta la subida más acusada $(84,4 €)$, siendo más del doble del incremento medio esperado. La razón que lo justifica es relativa a las características de las habitaciones. Si éstas tienen cabida para cuatro personas, significa que son más espaciosas y cuentan con mejores instalaciones y/o servicios incluidos, hechos que sin duda están siendo considerado por los hoteles durante la fijación de los precios (por ejemplo, habitaciones de tipo superior plus con cocina, junior suites con acceso al spa, pent-house suites con varias habitaciones y acceso al executive lounge, etc.).

La diferencia entre los precios medios por huésped de los extremos es relativamente pequeña $(163,0 €)$ si la comparamos con la existente entre el precio mínimo y máximo de la muestra (de $32,4 €$ a $2.500 €$ ). Esto, unido a que el precio medio no tiene incrementos proporcionales al número de huéspedes, puede explicar por qué los establecimientos concentran su oferta en habitaciones para tres o menos huéspedes $(96.5 \%)$. Los hoteleros en Sevilla que realizan un esfuerzo al dedicar más infraestructura a habitaciones que puedan albergar a cuatro personas y ofreciendo servicios premium, obtienen precios medios más altos, pero quizás no en consonancia. 
En línea con la idea anterior, si observamos el precio medio pagado por persona, veremos que éste disminuye a medida que la capacidad de la habitación va aumentando el número de personas a alojar (122,3€/pax en habitaciones de uso individual; 88,6€/pax en las dobles; $67,0 € /$ pax en las triples; y $71,3 € /$ en las cuádruples). En términos generales, cuantos más huéspedes se alojan en una habitación, menos debe pagar cada uno de ellos. Es cierto que, en el último caso, el precio pagado por persona se ve incrementado, pero el suplemento en el precio medio pagado por persona es marginal $(4,32 €)$.

Dicho incremento puede deberse a que, con frecuencia, el tipo de habitación que puede albergar la máxima capacidad (4 personas), se corresponde con habitaciones clasificadas como superior plus, junior suite y suite, las cuales se ofrecen a los precios más elevados $\mathrm{y}$, por tanto, el precio pagado por persona se incrementa en consecuencia. En cambio, las habitaciones que alojan a uno, dos o tres huéspedes normalmente están ligadas a tipologías estándar o superior en nuestra clasificación.

En cuanto a los precios mínimos publicados, los ofertados para tres o cuatro personas son más elevados que los precios medios de las categorías uno y dos huéspedes.

En este caso, la función exponencial es la que presenta un mejor nivel de ajuste $\left(\mathrm{R}^{2}=0,9695\right)$, lo que indica que los precios incrementan más rápido que el número de huéspedes de la habitación. El modelo estimado se presenta a continuación:

$$
\mathrm{y}=96,407 \mathrm{e}^{0.2666 x},
$$

(Expresión 4)

donde $\mathrm{x}$ indica el número de huéspedes que puede albergar la habitación $(\mathrm{x}=1,2,3,4)$.

\subsection{Longitud de estancia}

Si analizamos las variaciones de los precios medios bajo la influencia de la longitud de estancia (de una a cuatro noches) podemos concluir que los precios medios crecen a medida que el número de noches es mayor. Estos incrementos, en términos generales, no son proporcionales (Figura7). Los precios medios de cada categoría se expresan en precio por noche de hotel, no para el cómputo total de la estancia.

Por cada cambio ascendente en nivel de longitud de estancia, se espera un incremento medio de 37.1€. Los precios medios están aumentando en estas cantidades: $30,5 €$ (de una a dos noches), $31,4 €$ (de dos a tres noches) y 49,4€ (de tres a cuatro noches). Sólo en el último de los casos, los precios medios están aumentando en una cantidad superior al incremento medio esperado.

Cabría suponer que, en general, los hoteles podrían estar dispuestos a ofrecer precios por noche más bajos a condición de que sus clientes reserven una estancia más prolongada. En contrapartida, los clientes tendrían la expectativa opuesta, pagar un precio por noche más barato si se comprometen a permanecer en el hotel durante un número de noches concreto desde el momento de la reserva. Sin embargo, los datos revelan justo lo contrario.

En ciertos momentos, coincidiendo con festividades específicas a lo largo de todo el horizonte de reservas estudiado, los hoteles fijan precios por noche más altos con incrementos que llegan a alcanzar un 68,2\% (comparado el precio medio ofrecido para estancias de una noche con el correspondiente a cuatro noches). En estudios recientes, encontramos 
evidencias que vienen a refutar la misma conclusión, mostrando cómo hoteles en Estados Unidos fijan precios por noche más elevados cuando los huéspedes se alojan por más tiempo (Riasi, Schwarz, Liu y Li, 2017).

\section{Figura 7 \\ PRECIOS MEDIOS, MÁXIMOS Y MÍNIMOS POR LONGITUD DE ESTANCIA}

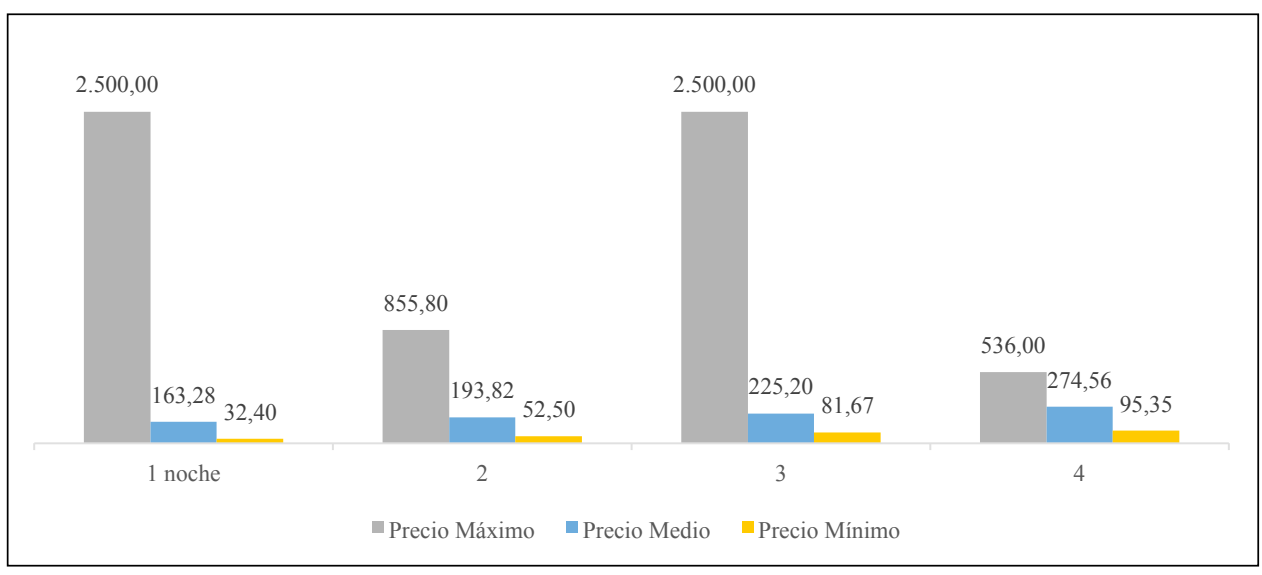

Otros datos son igualmente reseñables, por ejemplo, el precio noche máximo ofertado para una longitud de estancia de cuatro noches es el más bajo (536.0€). Estos valores máximos en los niveles de estancia anteriores (duración de la estancia, una, dos y tres noches) son $2.500,0 €, 855,8 €$ y $2.500,0 €$, respectivamente. En el caso de los precios mínimos registrados en cada nivel, estos van aumentando a medida que la longitud de estancia es también superior (los precios mínimos fijados varían entre $32.4 €$ y $95,35 €$ ).

Para finalizar, se ha comprobado que la función que representa más fielmente al comportamiento de los precios medios bajo la influencia de la duración de la estancia es de tipo exponencial (Expresión 5), $\mathrm{R}^{2}=0,9971$. El siguiente modelo estimado muestra un rápido comportamiento creciente de los precios medios conforme la longitud de la estancia se extiende en el tiempo:

$$
\mathrm{y}=137,18 \mathrm{e}^{0,1709 \mathrm{x}} \text {, }
$$

donde $\mathrm{x}$ indica la longitud de la estancia $(\mathrm{x}=1,2,3,4)$.

\section{CONCLUSIONES}

Este estudio explota un gran conjunto de datos de precios online ofertados en Booking. com por hoteles establecidos en Sevilla. Debido a que la investigación se ha llevado a cabo sobre una muestra representativa de hoteles de este destino, los resultados se pueden extrapolar a toda la población de hoteles (de tres, cuatro y cinco estrellas) de esta ciudad. 
En consecuencia, los revenue managers pueden usar los resultados de este estudio en su operativa y toma de decisiones, basándose en precios medios online publicados considerando una antelación 300 días, sin necesidad de llevar a cabo un análisis de precios exhaustivo y costoso en términos de tiempo. Los avances en el estudio de estos datos con la suficiente antelación podrán ayudar a los profesionales del sector hotelero a mejorar su gestión de precios de forma que consigan unos niveles de rentabilidad más elevados.

La gestión de canales de distribución es de una gran importancia en el actual panorama online de la distribución hotelera. La magnitud de los precios online ofrecidos por la muestra (193.393 precios publicados en un día por tan sólo 31 hoteles) puede aumentar a cifras aún superiores si pensamos en Sevilla como destino. Por ejemplo, un total de 94 hoteles de las categorías superiores en Sevilla tendría la capacidad de producir más de 580.000 precios ofertados por día, sólo en uno de sus canales de distribución online. Estas cifras representan una importante carga de trabajo, en cuanto a la cantidad de tiempo necesario para actualizar las tarifas y otras actividades relacionadas, por lo que la gestión de los canales de distribución se presenta cada vez más como un área principal a tener en cuenta.

Los resultados muestran que los precios aplicados a lo largo de todo el periodo de antelación varían según la categoría de hotel, el día de la semana, el tipo de habitación, el número de huéspedes máximo que pueden albergar las habitaciones y la duración de la estancia. También se confirma su vinculación con la literatura, ya que los precios medios aumentan con la categoría de hotel y con el número de huéspedes por habitación. En el caso del número de habitaciones, se ha comprobado que tiene influencia sobre los precios, pero sin presentar una pauta concreta.

Este estudio presenta algunas limitaciones, aunque al mismo tiempo establece líneas de investigación futuras. En primer lugar, se analizan los datos de un solo canal de distribución, aunque está reconocido como el más importante a nivel europeo.

En segundo lugar, se realiza un análisis agregado por variable, pero, ya que se ha comprobado que los precios muestran diferencias en relación con la categoría de hotel, se podría explorar su comportamiento en base a variables principales (día de la semana, tipo de habitación, número de huéspedes y duración de la estancia) según cada una de las categorías de hotel.

En tercer lugar, sería interesante estudiar el comportamiento de los precios con respecto al número de días que faltan hasta la fecha de check-in. Estudios previos prueban una tendencia ascendente en los precios online a medida que la fecha de cierre se acerca, pero sería de utilidad estudiar cómo es esta relación en el caso de Sevilla, por ejemplo, para diferentes categorías de hotel, tipos de habitación, duraciones de la estancia y temporada. Por último, el estudio en cualquiera de estos términos podría realizarse en otros destinos estableciendo comparativas, en lugar de explorar un único destino en detalle.

\section{AGRADECIMIENTOS}

Nos gustaría agradecer la participación de la empresa Beonprice S.L. en esta investigación, al Vicerrectorado de Investigación de la Universidad de Sevilla por la financiación del contrato de investigación de Sánchez-Lozano con cargo al V Plan Propio de Investigación; y al Centro de Investigação em Turismo, Sustentabilidade e Bem-estar (CinTurs) 
- Universidade do Algarve por su apoyo a esta investigación [Número de subvención FCT UID/SOC/04020/2020].

\section{BIBLIOGRAFÍA}

BAKER, T.K. y COLLIER, D.A. (1999): “A comparative revenue analysis of hotel yield management heuristics”, Decision Sciences, vol. 30 (1), pp. 239-263.

CHÁVEZ-MIRANDA, E. (2005): Yield management. Estudio de su aplicación en el sector hotelero. Tesis Doctoral. Universidad de Sevilla.

CHIANG, W., CHEN, J.C.H. y XU, X. (2007):“An overview of research on revenue management: current issues and future research", International Journal of Revenue Management, vol. 1 (1), pp. 97-128.

DOMINGO-CARRILLO, M.Á. (2016): Investigación sobre revenue management en turismo en el sector hotelero. Estudio empírico sobre hoteles de 4 y 5 estrellas. Tesis Doctoral. Universidad de Sevilla.

DOMINGO-CARRILLO, M.Á., CHÁVEZ-MIRANDA, E. y CUBILES-DE LA VEGA, M.D. (2017): "Journal segmentation and competitive position based on revenue management research publications", Journal of Revenue and Pricing Management, vol. 16 (5), pp. 466-482.

EXCELTUR (2013): UrbanTUR 2012. Monitor de competitividad turística de los destinos urbanos españoles. Madrid, Exceltur.

EXCELTUR (2016): Barómetro de la rentabilidad y el empleo de los destinos turísticos españoles. Balance 2015. Madrid, Exceltur.

EXCELTUR (2017): Barómetro de la rentabilidad y el empleo de los destinos turísticos españoles. Balance 2016. Madrid, Exceltur.

EXCELTUR (2017):UrbanTUR 2016. Monitor de competitividad turística de los destinos urbanos españoles. Madrid: Exceltur.

EXCELTUR (2018): Barómetro de la rentabilidad y el empleo de los destinos turísticos españoles. Balance 2017. Madrid, Exceltur.

GUILLET, B.D. y MOHAMMED, I. (2015) : "Revenue management research in hospitality and tourism: A critical review of current literature and suggestions for future research", International Journal of Contemporary Hospitality Management, vol. 27 (4), pp. 526-560.

IVANOV, S. y ZHECHEV, V. (2012): "Hotel revenue management-a critical literature review”. Turizam: znanstveno- stručni časopis, vol. 60 (2), pp. 175-197.

JOSEPHI, S.H.G., STIERAND, M.B. y VAN MOURIK, A. (2016): "Hotel revenue management: Then, now and tomorrow", Journal of Revenue and Pricing Management, vol. 15 (3-4), pp. 252-257.

KIMES, S.E. (2016): "The evolution of hotel revenue management", Journal of Revenue and Pricing Management, vol. 15 (3-4), pp. 247-251.

LAW, R., LEUNG, R., LO, A., LEUNG, D., HOC, L. y FONG, L.H.N. (2015): “Distribution channel in hospitality and tourism: Revisiting disintermediation from the perspectives of hotels and travel agencies", International Journal of Contemporary Hospitality Management, vol. 27 (3), pp. 431-452. 
LONELY PLANET (2018): “Best in travel 2018: las 10 mejores ciudades”. Consultado 19 abril, 2018. https:/www.lonelyplanet.es/blog/best-in-travel-2018-las-10-mejores-ciudades

MCGILL, J.I. y VAN RYZING, G.J. (1999): "Revenue management: Research overview and prospects", Transportation Science, vol. 33 (2), pp. 233-256.

OTEO-ITURMENDI, J.M. (2013): La aplicación del yield management en el sector hotelero: El caso de los hoteles de las tres capitales vascas. Tesis Doctoral. Universidad de Deusto.

PAWLICZ, A. y NAPIERALA, T. (2017): “The determinants of hotel room rates: An analysis of the hotel industry in Warsaw, Poland", International Journal of Contemporary Hospitality Management, vol. 29 (1), pp. 571-588.

RIASI, A., SCHWARTZ, Z., LIU, X. y LI, S. (2017): "Revenue management and lengthof-stay-based room pricing", Cornell Hospitality Quarterly, vol. 58 (4), pp. 393-399.

ROPERO-GARCÍA, M.Á. (2011): "Dynamic pricing policies of hotel establishments in an online travel agency", Tourism Economics, vol. 17 (5), pp. 1.087-1.102.

TALÓN-BALLESTERO, M.P. (2010): Revenue yield management en los hoteles de Madrid: Análisis empírico de su aplicación y resultados. Tesis Doctoral. Universidad Rey Juan Carlos. Madrid.

TOM DIECK, M. C., FOUNTOULAKI, P. y JUNG, T. H. (2018): “Tourism distribution channels in European island destinations", International Journal of Contemporary Hospitality Management, vol. 30 (1), pp. 326-342.

VINOD, B. (2004): "Unlocking the value of revenue management in the hotel industry", Journal of Revenue and Pricing Management, vol. 3 (2), pp. 178-190.

WEATHERFORD, L. y BODILIY, S.E. (1992): "A taxonomy and research overview of perishable-asset revenue management: Yield management, overbooking and pricing", Operations Research, vol. 40 (5), pp. 831-844. 